\title{
The Outcomes of Tracheostomy in Pediatric Cardiac Surgical Patients
}

\author{
Manoj Kumar Sahu ${ }^{1}$ Challatil Bipin ${ }^{1} \quad$ Ummed Singh Dhatterwal ${ }^{1}$ Sarvesh Pal Singh ${ }^{1}$ \\ Velayoudam Devagourou² Palleti Rajashekar ${ }^{2, \odot ~}$ Sachin Talwar $^{2}$ \\ Milind Padmakar Hote ${ }^{2}$ Shiv Kumar Chaudhary ${ }^{2}$
}

${ }^{1}$ Department of Cardiothoracic and Vascular Surgery, Intensive Care for CTVS, Cardiothoracic and Neurosciences Centre, All India Institute of Medical Sciences, Ansari Nagar, New Delhi, India

2Department of Cardiothoracic and Vascular Surgery,

Cardiothoracic and Neurosciences Centre, All India Institute of Medical Sciences, Ansari Nagar, New Delhi, India52124133

\begin{abstract}
Address for correspondence Manoj Kumar Sahu, MD, DNB, Department of CTVS, Intensive Care for CTVS, Room No 5, $7^{\text {th }}$ floor, Cardiothoracic and Neurosciences Centre, All India Institute of Medical Sciences, New Delhi, 110029, India

(e-mail: drmanojsahu@gmail.com).
\end{abstract}

J Card Crit Care 2021;5:124-133.

Abstract

Keywords
- Pediatric cardiac
surgery
- Intensive care
- Tracheostomy
- Prolonged mechanical
ventilation

Objectives To describe the outcomes of postoperative tracheostomy and determine the predictors of survival.

Design Prospective, observational study.

Setting Cardiac surgical intensive care unit of a tertiary care hospital.

Participants All pediatric patients below 10 years of age who underwent tracheostomy after cardiac surgery from January 2019 to December 2019. Different variables were compared between survivors and nonsurvivors.

Intervention Tracheostomy.

Results Among 1084 pediatric patients who underwent cardiac surgery during the study period, 41 (3.7\%) received tracheostomy. Survival rate was $71 \%$.

Earlier, sternal closure (SC) $(p=0.04)$, acute kidney injury (AKI) $(p=0.001)$, serum C-reactive protein (CRP) $(p=0.007)$, duration of total parenteral nutrition (TPN) $(p=0.005)$ and days of feed interruption $(\mathrm{FI})(p=0.02)$, activated partial thromboplastin time (aPTT) before tracheostomy $(p=0.006)$, and bleeding from tracheostomy site $(p=0.02)$ were significantly low in the survivor group.

Among the peritracheostomy variables taken at different time points, the levels of $\mathrm{pH}$ on tracheostomy day $1(p=0.03)$, serum lactate on tracheostomy day $2(p=0.01)$ and day $3(p=0.01)$, and random blood sugar (RBS) on tracheostomy day $3(p=0.04)$ were significantly lower in the survivor group.

The arterial oxygen saturation $(\mathrm{SaO} 2)$ on tracheostomy day $1(p=0.04)$ and the platelet count before tracheostomy $(p=0.02)$ were significantly higher in the survivor group.

Conclusions Our study demonstrated a survival of $71 \%$ among the study cohort. Lesser duration of open sternum, lower incidence of AKI, less number of days on TPN and FI, lower posttracheostomy aPTT, bleeding, RBS, lactate, and higher pretracheostomy platelet count and posttracheostomy $\mathrm{SaO} 2$ were found to be the predictors of survival.
DOI https://doi.org/

10.1055/s-0041-1732841

ISSN 2457-0206
(C) 2021. Official Publication of The Simulation Society (TSS), accredited by International Society of Cardiovascular Ultrasound (ISCU). This is an open access article published by Thieme under the terms of the Creative Commons Attribution-NonDerivative-NonCommercial-License, permitting copying and reproduction so long as the original work is given appropriate credit. Contents may not be used for commercial purposes, or adapted, remixed, transformed or built upon. (https://creativecommons.org/licenses/by-nc-nd/4.0/).

Thieme Medical and Scientific Publishers Pvt. Ltd. A-12, 2nd Floor, Sector 2, Noida-201301 UP, India 


\section{Introduction}

Congenital cardiac surgeries in infants and children have taken a giant leap in the last couple of decades, and an increasing number of complex surgeries are being performed around the world. ${ }^{1}$ Innumerable children require prolonged postoperative care in terms of longer mechanical ventilation (MV), inotropic support, and nutritional care. It has been found that tracheostomy helps in smoother weaning from $\mathrm{MV}$, reducing the incidence of sepsis, better enteral feeding, and overall postoperative convalescence. Tracheostomy as a cardiorespiratory support is indicated in some of these children, with high-risk surgical procedure for complex congenital cardiac diseases, syndromic children, airway issues and cardiac-related comorbidities such as severe ventricular dysfunction, low-cardiac output, residual lesions, sepsis related to prolonged MV, etc. ${ }^{1}$

The incidence of tracheostomy after pediatric cardiac operations in various studies presented ranged from $0.2 \%$ to $2.7 \% .^{1}$ A retrospective study including over 4000 patients from the Society of Thoracic Surgeons (STS) database concluded that patients who underwent tracheostomy, had lesser survival than those without tracheostomy. However, the other single-center retrospective reports studied relatively small number of patients, and demonstrated a mortality ranging from 0 to $40 \%$ in pediatric cardiac surgical patients who underwent tracheostomy. ${ }^{1-6}$ Although the procedure looks infrequent from the existing studies, tracheostomy is being looked upon as a major support system in the continuing care of these children postoperatively. We did not find any prospective study on outcomes of tracheostomy in this subset of patients. We thus decided to evaluate the survival, the predictors of survival, and other outcomes such as length of intensive care unit (ICU) and hospital stay and total duration of MV among the postoperative pediatric cardiac surgical patients.

\section{Methods}

We conducted a prospective study on 1084 children $\leq$ 10 years of age who underwent cardiac surgery during the study period from January 2019 to December 2019 at a tertiary care institute and were under care at our cardiac surgical intensive care unit (CSICU). A total of 41 of them underwent tracheostomy postoperatively.

This study was approved by the Institute Ethics Committee. Informed consent was taken from parents of the participants. Those who were tracheostomized before surgery as well as those who did not wish to participate were excluded from the study.

The decision to perform tracheostomy was made by the intensivist and cardiac surgeon together, based on postoperative problems like cardiac complications, including ventricular dysfunction, residual defects, neurological injury, sepsis, airway abnormality, inability to wean from MV, etc.

The timing of tracheostomy was based on the hemodynamic stability, the anticipated course in the ICU including morbidities and, after reviewing, various clinical and laboratory parameters. Tracheostomy was considered to be early if performed within 7 days of onset of MV.

All the tracheostomies were performed surgically at the bedside in the CSICU. Enteral feeding was interrupted for 6 hours for the procedure. Choice of cuffed or uncuffed tube was based on the standard guidelines as per age of the patient. Antibiotics were continued as per the unit protocol, based on individual patient profile. Posttracheostomy weaning of MV was continued in a stepwise manner. Enteral nutrition was continued through Ryle's tube till child started accepting orally. Patients were discharged from ICU to the ward after a mandatory period of 48 hours of unsupported ventilation, minimal secretions, and adequate gas exchange. We preferably tried to decannulate our patients in the ICU before discharge.

Data of the study participants including preoperative, intraoperative, postoperative, peritracheostomy, and outcome variables were collected and analyzed. These data were compared between the survivors and nonsurvivors. The outcomes of the study were survival, the predictors of survival, and other outcomes like the timing of tracheostomy, total days of MV, length of ICU stay (LOICUS) and length of hospital stay (LOHS).

\section{Definitions}

1. Underweight, wasting, and stunting were defined, according to the National Centre for Health Statistics (NCHS) Standards classification.?

2. High-risk cardiac surgery was defined, according to the risk adjustment for congenital heart surgery (RACHS) 1 score.

3. Acute kidney injury (AKI) was defined, according to the kidney disease improving global outcomes (KDIGO) criteria. $^{8}$

4. Central nervous system (CNS) event: Any change in the Glasgow coma scale (GCS) score or any postoperative neurologic disorder (visual, cognitive, motor, or speech) documented and/or neurologic disability severely affecting day-to-day functioning was attributed to a CNS pathology or biochemical changes.

5. Sepsis was defined as per the sepsis 3 definition. ${ }^{9}$

6. Hospital-acquired infections (HAI), including catheter-associated urinary tract infection (CAUTI), catheter-related blood stream infection (CRABSI), ventilator-associated pneumonia (VAP), and surgical site infection (SSI) were defined, according to the center for disease control and prevention (CDC) definitions. ${ }^{10}$

\section{Statistical Analysis}

Statistical analysis was performed with the use of SPSS version 20 (Stata Corp, 4905, Lake way Drive, College Station, Texas, 77845 , USA). Demographic and clinical variables were described as mean \pm standard deviation (SD) for normally distributed continuous data, median (interquartile range [IQR]) for skewed data, and frequencies (\%) for categorical variables. Variables were compared using nonparametric WilcoxonRank sum test for the continuous data and Fisher's exact test 
for categorical variables. A " $p$ " value $<0.05$ was considered statistically significant.

\section{Results}

During the 1-year study period, 1084 index pediatric cardiac operations were performed on children below 10 years of age. Tracheostomy was performed in 41 (3.7\%) of these patients.

Details regarding comprehensive cardiac diagnoses, surgical procedures, RACHS1 score, and survival in the study population are elaborated in - Table 1. Patient demography, anthropometric measurements, and pre and intraoperative variables are compared in -Table 2 .

The mean age of the 41 tracheostomized children was $3 \pm$ 4.1 years (median 1 year). As many as 30 (73\%) of these children were males and 11 (27\%) were females. Five children were syndromic-two had trisomy 21 , one child had thrombocytopenia absent radius (TAR) syndrome, one had Goldenhar syndrome, and one had Vertebral defect, Anal atresia, Cardiac defect, Tracheoesophageal fistula, Renal and Limb abnormality (VACTERL) association. Preoperative variables such as sepsis, MV, previous surgery, chronic lung disease, neuromuscular disease, etc. were assessed among the study population.
Ten (24.3\%) children with transposition of great arteries (TGA) underwent arterial switch operation (ASO), and they constituted the majority of the tracheostomized patients. The primary outcome of our study, survival, was $71 \%$.

Postoperative complications such as bleeding, reexploration, extracorporeal membrane oxygenation (ECMO) requirement, pressure sores, unanticipated cardiac arrest, accidental tracheostomy tube dislodgement, CNS injury, AKI, etc. were compared between survivors and nonsurvivors in - Table 3. Among the various postoperative complications, delayed sternal closure (SC) was the major morbidity (63\%), while ECMO requirement (4.8\%) was the least common complication.

About $31.7 \%$ of the children developed sepsis. VAP formed the major subset of HAI, while CAUTI was the least in this cohort. Variables pertaining to HAI were compared in - Table 4. We evaluated the variables pertaining to postoperative nutrition such as duration of NPO (nil per oral), days on total parenteral nutrition (TPN), days of enteral feed and feed interruption (FI), and these variables were compared between survivors and nonsurvivors in - Table 5.

Postoperative outcome variables like duration of MV, tracheostomy complications, reasons for prolonged MV, LOICUS,

Table 1 Pediatric cardiac surgery patients who received tracheostomy postoperatively

\begin{tabular}{|c|c|c|c|c|c|}
\hline Sl. no. & Diagnosis & Cardiac surgery & Patients, $n$ (\%) & RACHS 1 Score & Survivors, $n(\%)$ \\
\hline 1 & TOF & Total correction & $9(23.2)$ & 2 & $6(66.6)$ \\
\hline 2 & TOF with Pulmonary atresia (PA) & Conduit repair & $1(2.4)$ & 2 & $1(100)$ \\
\hline 3 & d- TGA & $\begin{array}{l}\text { Arterial switch opera- } \\
\text { tion (ASO) }\end{array}$ & $10(24)$ & 4 & $7(70)$ \\
\hline 4 & VSD & VSD closure & $3(7.2)$ & 2 & $3(100)$ \\
\hline 5 & SC TAPVC & TAPVC repair & $2(4.8)$ & 2 & $2(100)$ \\
\hline 6 & IC TAPVC & TAPVC repair & $2(4.8)$ & 4 & $0(0)$ \\
\hline 7 & AVSD & AVSD repair & $1(2.4)$ & 3 & $1(100)$ \\
\hline 8 & AVSD PAH & AVSD repair & $4(9.6)$ & 4 & $4(100)$ \\
\hline 9 & Partial AVSD PDA severe PAH & $\begin{array}{l}\text { PA band atrial septec- } \\
\text { tomy PDA ligation }\end{array}$ & $1(2.4)$ & 3 & $0(0)$ \\
\hline 10 & Ebstein anomaly & Cone repair & $1(2.4)$ & 5 & $1(100)$ \\
\hline 11 & PA IVS & $\begin{array}{l}\text { Pulmonary valvotomy } \\
\text { RVOT patch }\end{array}$ & $1(2.4)$ & 2 & $1(100)$ \\
\hline 12 & Truncus arteriosus (type 3) & $\begin{array}{l}\text { Truncus arteriosus } \\
\text { repair }\end{array}$ & $1(2.4)$ & 5 & $0(0)$ \\
\hline 13 & AP window & AP window repair & $1(2.4)$ & 2 & $1(100)$ \\
\hline 14 & IPAH severe PAH & Pott shunt & $1(2.4)$ & 3 & $1(100)$ \\
\hline 15 & $\begin{array}{l}\text { TOF with PA hypoplastic main and } \\
\text { left pulmonary arteries. }\end{array}$ & $\begin{array}{l}\text { RMBTS followed by BD } \\
\text { GLENN }\end{array}$ & $1(2.4)$ & 3 & $1(100)$ \\
\hline 16 & VSD mesocardia small RV & BD GLENN & $1(2.4)$ & 2 & $0(0)$ \\
\hline 17 & $\begin{array}{l}\text { d-TGA large inlet VSD, PA, } \\
\text { aortopulmonary collaterals post } \\
\text { RMBTS/BD GLENN / azygous vein } \\
\text { ligation post coil }\end{array}$ & $\begin{array}{l}\text { Completion Fontan } \\
\text { Operation }\end{array}$ & $1(2.4)$ & 3 & $0(0)$ \\
\hline & Total & & $41(100)$ & & $29(71)$ \\
\hline
\end{tabular}

Abbreviations: AP, aortopulmonary; ASO, arterial switch operation; AVSD, atrioventricular Septal Defect; BD, bidirectional; IC, infracardiac; IPAH, idiopathic pulmonary artery hypertension; IVS, intact ventricular septum; PA, pulmonary atresia; PAH, pulmonary artery hypertension; PDA, patent ductus arteriosus; RACHS, risk adjustment for congenital heart surgery; RMBTS, right modified Blalock-Taussig shunt; RV, right ventricle; RVOT, right ventricular outflow tract; SC, supracardiac; TAPVC, total anomalous pulmonary venous connection; TCA, transposition of great arteries; TOF, tetralogy of Fallot; VSD, ventricular septal defect. 
Table 2 Comparison of the preoperative and intraoperative characteristics between survivors and nonsurvivors

\begin{tabular}{|c|c|c|c|c|}
\hline SI. no. & Clinical variables & Survivors $(n=29)$ & Nonsurvivors $(n=12)$ & $p$-value \\
\hline 1 & Age in years (mean $\pm S D$ ) & $2.82 \pm 4.27$ & $3.46 \pm 3.66$ & 0.75 \\
\hline 2 & $\begin{array}{l}\text { Age categories, } n(\%) \\
\leq 1 \text { years } \\
>1 \text { to } 5 \\
>5 \text { to } 10 \text { years }\end{array}$ & $\begin{array}{l}15(51.7) \\
10(34.6) \\
4(13.7)\end{array}$ & $\begin{array}{l}4(33.3) \\
4(33.3) \\
4(33.3)\end{array}$ & 0.06 \\
\hline 4 & Male, $n(\%)$ & $22(75.8)$ & $8(66.6)$ & 0.40 \\
\hline 5 & Weight in $\mathrm{kg}$ (mean \pm SD) & $9.22 \pm 7.64$ & $9.08 \pm 6.15$ & 0.94 \\
\hline 6 & Underweight, $n(\%)$ & $3(10.3)$ & $2(16.6)$ & 0.38 \\
\hline 7 & Height in cm (mean \pm SD) & $80.27 \pm 27.29$ & $84.08 \pm 29.41$ & 0.72 \\
\hline 8 & Stunting, $n(\%)$ & $16(55)$ & $5(41.6)$ & 0.57 \\
\hline 9 & Wasting, $n(\%)$ & $6(20.6)$ & $1(8.3)$ & 0.44 \\
\hline 10 & Preop hospitalization in days (mean \pm SD) & $7.55 \pm 12.4$ & $8.5 \pm 8.8$ & 0.08 \\
\hline 11 & Chromosomal anomaly, n (\%) & $3(10.3)$ & $2(16.6)$ & 0.46 \\
\hline 12 & Neuromuscular disorder, $n(\%)$ & $1(3.4)$ & $0(0)$ & 0.72 \\
\hline 13 & Chronic lung disease, $n$ (\%) & $0(0)$ & $1(8.3)$ & 0.29 \\
\hline 14 & Preop MV, n (\%) & $3(10.3)$ & $2(16.6)$ & 0.46 \\
\hline 15 & Previous surgery, $n$ (\%) & $6(20.6)$ & $3(25)$ & 0.53 \\
\hline 16 & Preop sepsis, $n$ (\%) & $7(24.1)$ & $3(25)$ & 0.62 \\
\hline 17 & Cyanotic, n (\%) & $24(82.7)$ & $9(75)$ & 0.43 \\
\hline 18 & Increased Qp, n (\%) & $14(48.3)$ & $6(50)$ & 0.634 \\
\hline 19 & Univentricular heart, $n$ (\%) & $2(6.8)$ & $2(16.6)$ & 0.332 \\
\hline 20 & High risk surgery, n (\%) & $12(41.3)$ & $6(50)$ & 0.73 \\
\hline 21 & Cardiopulmonary bypass time in minutes (mean \pm SD) & $167.69 \pm 99.03$ & $151.5 \pm 90.1$ & 0.54 \\
\hline 22 & Aortic cross clamp time in minutes (mean \pm SD) & $108.36 \pm 65.5$ & $97.18 \pm 65.26$ & 0.46 \\
\hline
\end{tabular}

$\mathrm{MV}$, mechanical ventilation; $\mathrm{n}$, number of; Qp, pulmonary blood flow; SD, standard deviation.

Table 3 Comparison of postoperative complications between survivors and nonsurvivors

\begin{tabular}{|c|c|c|c|c|}
\hline SI. no. & Clinical variables & Survivors $(n=29)$ & Non survivors $(n=12)$ & $p$-value \\
\hline 1 & Major bleeding, $n(\%)$ & $9(31)$ & $3(25)$ & 0.50 \\
\hline 2 & Postoperative PRBC transfusion in $\mathrm{mL} / \mathrm{kg}$ (mean \pm SD) & $51.08 \pm 40.16$ & $96.24 \pm 106.32$ & 0.32 \\
\hline 3 & Postoperative FFP transfusion in $\mathrm{mL} / \mathrm{kg}$ (mean \pm SD) & $38.32 \pm 25.97$ & $38.57 \pm 10.30$ & 0.64 \\
\hline 4 & Postoperative platelet transfusion in $\mathrm{mL} / \mathrm{kg}$ (mean $\pm \mathrm{SD}$ ) & $25.00 \pm 25.46$ & $65.76 \pm 114.51$ & 0.21 \\
\hline 5 & Reexploration, $n(\%)$ & $17(58.6)$ & $7(58.3)$ & 0.78 \\
\hline 6 & $\begin{array}{l}\text { Causes of reexploration, } n \text { (\%) } \\
\text { Bleeding } \\
\text { Residual cardiac defect }\end{array}$ & $\begin{array}{l}8(27.6) \\
9(31)\end{array}$ & $\begin{array}{l}3(25) \\
4(33.3)\end{array}$ & 0.9 \\
\hline 7 & Delayed SC, $n$ (\%) & $20(70)$ & $6(50)$ & 0.21 \\
\hline 8 & Day of SC (mean \pm SD) & $1.4 \pm 1.07$ & $4.5 \pm 3.72$ & 0.0479 \\
\hline 9 & ECMO instituted, $n(\%)$ & $2(7)$ & $0(0)$ & 0.49 \\
\hline 10 & CNS events, $n(\%)$ & $7(24.1)$ & $5(41.6)$ & 0.22 \\
\hline 11 & $\begin{array}{l}\text { CNS events, } n(\%) \\
\text { Seizure } \\
\text { Low GCS }\end{array}$ & $\begin{array}{l}6(20.6) \\
1(3.5)\end{array}$ & $\begin{array}{l}4(33.3) \\
1(8.3)\end{array}$ & 0.68 \\
\hline 12 & AKI, $n(\%)$ & $5(17.2)$ & $11(91.6)$ & 0.001 \\
\hline 13 & Unanticipated arrest, $n$ (\%) & $9(31)$ & $6(50)$ & 0.21 \\
\hline 14 & Accidental tracheal tube dislodgement, $n(\%)$ & $4(13.8)$ & $2(17)$ & 0.57 \\
\hline 15 & Pressure sore, $n(\%)$ & $6(20.6)$ & $6(50)$ & 0.06 \\
\hline
\end{tabular}

Abbreviations: AKI, acute kidney injury; CNS, central nervous system; ECMO, extracorporeal membrane oxygenation; FFP, fresh frozen plasma; GCS, Glasgow coma scale; PRBC, packed red blood cells; SC, sternal closure; SD, standard deviation. 
Table 4 Comparison of postoperative variables pertaining to infection/sepsis between survivors and nonsurvivors

\begin{tabular}{|c|c|c|c|c|}
\hline SI. no. & Clinical variables & Survivors $(n=29)$ & Nonsurvivors $(n=12)$ & $p$-value \\
\hline 1 & Sepsis, $n(\%)$ & $8(27.5)$ & $5(41.6)$ & 0.46 \\
\hline 2 & $\begin{array}{l}\text { Pathogens isolated, } \boldsymbol{n}(\%) \\
\text { Pseudomonas } \\
\text { Pseudomonas + Acinetobacter } \\
\text { Klebsiella +S. maltophila } \\
\text { Klebsiella } \\
\text { E. coli + Acinetobacter } \\
\text { Proteus } \\
\text { S. maltophila } \\
\text { Acinetobacter } \\
\text { S. hemolyticus + Candida } \\
\text { Klebsiella + Pseudomonas }\end{array}$ & $\begin{array}{l}2(6.8) \\
1(3.4) \\
0(0) \\
2(6.8) \\
0(0) \\
0(0) \\
1(3.4) \\
0(0) \\
1(3.4) \\
1(3.4)\end{array}$ & $\begin{array}{l}0(0) \\
0(0) \\
1(8.3) \\
1(8.3) \\
1(8.3) \\
1(8.3) \\
0(0) \\
1(8.3) \\
0(0) \\
0(0)\end{array}$ & 0.36 \\
\hline 4 & $\mathrm{CRP}$ in $\mathrm{mg} / \mathrm{l}($ mean $\pm \mathrm{SD})$ & $56.65 \pm 54.98$ & $111.27 \pm 41.11$ & 0.007 \\
\hline 5 & $\mathrm{PCT}$ in $\mathrm{ng} / \mathrm{mL}($ mean $\pm \mathrm{SD})$ & $20.25 \pm 26.77$ & $42.42 \pm 53.11$ & 0.09 \\
\hline 6 & Galactomannan in ODI (mean \pm SD) & $1.23 \pm 0.34$ & $0.79 \pm 0.70$ & 0.23 \\
\hline 7 & VAP, n (\%) & $9(31.03)$ & $4(33.3)$ & 0.9 \\
\hline 8 & Pretracheostomy VAP, n (\%) & $5(17.2)$ & $3(25)$ & 0.67 \\
\hline 9 & Posttracheostomy VAP, n (\%) & $4(13.7)$ & $1(8.3)$ & 0.9 \\
\hline 10 & CRBSI, n (\%) & $1(3.4)$ & $1(8.3)$ & 0.50 \\
\hline 11 & CAUTI, n (\%) & $1(3.4)$ & $1(8.3)$ & 0.50 \\
\hline 12 & Tracheostomy site infection, $n(\%)$ & $4(13.7)$ & $4(33.3)$ & 0.15 \\
\hline 13 & SSI, $n(\%)$ & $2(6.8)$ & $2(8.6)$ & 0.33 \\
\hline 14 & Antibiotic escalation needed, $n(\%)$ & $15(52)$ & $9(75)$ & 0.29 \\
\hline 15 & Antifungal therapy required, $n$ (\%) & $11(38)$ & $4(33.3)$ & 0.65 \\
\hline
\end{tabular}

Abbreviations: CAUTI, catheter-associated urinary tract infection; CRBSI, catheter-related blood stream infection; CRP, C-reactive protein; ODI, optical density index; PCT, procalcitonin; SD, standard deviation; SSI, surgical site infection; VAP, ventilator-associated pneumonia.

Table 5 Comparison of postoperative clinical variables pertaining to nutrition between survivors and nonsurvivors

\begin{tabular}{|l|l|l|l|l|}
\hline SI. no. & Clinical variables & Survivors $(\boldsymbol{n}=\mathbf{2 9})$ & Nonsurvivors $(\boldsymbol{n}=12)$ & $p$-value \\
\hline 1 & Days on NPO (mean \pm SD) & $2.44 \pm 2.54$ & $3.75 \pm 4.04$ & 0.19 \\
\hline 2 & Days on TPN (mean \pm SD) & $2.71 \pm 5.32$ & $10.87 \pm 8.69$ & 0.0056 \\
\hline 3 & Days of FI (mean \pm SD) & $4.28 \pm 4.35$ & $10.91 \pm 8.91$ & 0.0270 \\
& Categories of FI ( $\boldsymbol{n})(\%)$ & $0(0)$ & $1(8.3)$ & 0.08 \\
& Seizure & $4(13.6)$ & $2(16.6)$ & \\
& Surgical procedure & $9(30.6)$ & $1(8.3)$ & \\
& Feed intolerance & $9(30.6)$ & $3(24.5)$ & \\
& Surgical procedure+ feed intolerance & $1(3.4)$ & & \\
& Seizure+ feed intolerance & & & \\
\end{tabular}

Abbreviations: Fl, feed interruption; NPO, nil per oral; SD, standard deviation; TPN, total parenteral nutrition.

and LOHS were described in - Table 6. Many perioperative variables like open sternum duration, AKI, C-reactive protein (CRP), days of TPN and days of FI, activated partial thromboplastin time (aPTT) before tracheostomy, bleeding from tracheostomy site, the day of tracheostomy initiation, days of MV with tracheostomy, total days of MV, number of extubation trials, tracheal complications, etc. were compared between survivors and nonsurvivors. The major indication for tracheostomy was prolonged MV due to cardiac causes. Peritracheostomy variables including hemodynamics, arterial blood gas (ABG), laboratory parameters, positive end expiratory pressure (PEEP) requirement, and bleeding from the tracheostomy site were compared in $\boldsymbol{\sim}$ Table 7.
The day of SC $(p=0.04)$, AKI $(p=0.001)$, serum C-reactive protein (CRP) levels $(p=0.007)$, days of TPN $(p=0.005)$ and days of FI ( $p=0.02)$, aPTT before tracheostomy $(p=0.006)$, and bleeding from tracheostomy site $(p=0.02)$ were significantly low in the survivor group. Among the peritracheostomy variables taken at different time points, $\mathrm{pH}$ on tracheostomy day $1(p=0.03)$, serum lactate on tracheostomy day $2(p=0.01)$ and day 3 ( $p=0.01$ ), and random blood sugar (RBS) on tracheostomy day $3(p=0.04)$ were significantly lower in the survivor group. The arterial oxygen saturation $(\mathrm{SaO} 2)$ on tracheostomy day $1(p=0.04)$ and the platelet count before tracheostomy $(p=0.02)$ were significantly higher in the survivor group. Some of the outcome variables such as mortality, total 
Table 6 Comparison of postoperative outcome variables between survivors and nonsurvivors

\begin{tabular}{|c|c|c|c|c|}
\hline SI. no. & Clinical variables & Survivors $(n=29)$ & Nonsurvivors $(n=12)$ & $p$-value \\
\hline 1 & Day of tracheostomy from MV initiation (mean \pm SD) & $11.41 \pm 7.46$ & $16.75 \pm 15.96$ & 0.37 \\
\hline 2 & Duration of endotracheal tube MV in days (mean \pm SD) & $11.5 \pm 7.08$ & $16.75 \pm 15.96$ & 0.44 \\
\hline 3 & Duration of tracheostomy tube MV in days (mean \pm SD) & $19.27 \pm 18.22$ & $26.5 \pm 21.43$ & 0.36 \\
\hline 4 & Total duration of MV in days (mean \pm SD) & $30.34 \pm 21.21$ & $42.41 \pm 27.38$ & 0.19 \\
\hline 5 & No. of extubation trails (mean \pm SD) & $1.24 \pm 0.91$ & $0.75 \pm 0.96$ & 0.12 \\
\hline 6 & No. of times TT changed (mean \pm SD) & $2 \pm 2.81$ & $1.25 \pm 1.35$ & 0.66 \\
\hline 7 & $\begin{array}{l}\text { Reasons for TT change, } \boldsymbol{n}(\%) \\
\text { TT block } \\
\text { Routine downsizing of TT } \\
\text { Accidental dislodgement of TT } \\
\text { Miscellaneous reasons }\end{array}$ & $\begin{array}{l}3(10.2) \\
4(13.6) \\
2(6.8) \\
9(30.6)\end{array}$ & $\begin{array}{l}1(8.4) \\
1(8.4) \\
0(0) \\
5(41.5)\end{array}$ & 0.88 \\
\hline 8 & LOICUS in days (mean \pm SD) & $38.03 \pm 24.92$ & $43.75 \pm 27.73$ & 0.70 \\
\hline 9 & LOHS in days (mean \pm SD) & $55 \pm 37.42$ & $54.83 \pm 35.90$ & 0.87 \\
\hline 10 & Total MV-free days (mean \pm SD) & $25.75 \pm 26.09$ & $27.75 \pm 25.96$ & 0.80 \\
\hline 11 & Duration of tracheal cannulation in days (mean \pm SD) & $32.34 \pm 38.97$ & $29.41 \pm 28.06$ & 0.7963 \\
\hline 12 & Tracheostomy complications, $n$ (\%) & $8(27.5)$ & $5(41.6)$ & 0.300 \\
\hline 13 & $\begin{array}{l}\text { Tracheostomy complications, } n \text { (\%) } \\
\text { Tracheal stenosis } \\
\text { TBM } \\
\text { Granulation } \\
\text { Tracheal rent }\end{array}$ & $\begin{array}{l}5(17.3) \\
1(3.4) \\
2(6.8) \\
0(0)\end{array}$ & $\begin{array}{l}1(8.3) \\
0(0) \\
3(33) \\
1(8.3)\end{array}$ & $>0.05$ \\
\hline 14 & $\begin{array}{l}\text { Indication for tracheostomy, } n \text { (\%) } \\
\text { Prolonged MV } \\
\text { Smooth weaning from MV }\end{array}$ & $\begin{array}{l}21(32.5) \\
8(27.5)\end{array}$ & $\begin{array}{l}11(91.7) \\
1(8.3)\end{array}$ & 0.23 \\
\hline 15 & $\begin{array}{l}\text { Reason for prolonged MV, } n \text { (\%) } \\
\text { Cardiac } \\
\text { Cardiac + diaphragmatic palsy } \\
\text { Cardiac + CNS event+ chylothorax } \\
\text { Cardiac + CNS event } \\
\text { Cardiac + chylothorax } \\
\text { Cardiac + SCS } \\
\text { Cardiac +SGS +CNS event }\end{array}$ & $\begin{array}{l}7(23.8) \\
1(3.4) \\
1(3.4) \\
4(13.6) \\
3(10.2) \\
4(13.6) \\
1(3.4)\end{array}$ & $\begin{array}{l}5(41.5) \\
0(0) \\
0(0) \\
4(33.3) \\
1(8.3) \\
1(8.3) \\
0(0)\end{array}$ & 0.824 \\
\hline
\end{tabular}

Abbreviations: CNS, central nervous system; ICU, intensive care unit; LOHS, length of hospital stay; LOICUS, length of ICU stay; MV, mechanical ventilation; n, number; SD, standard deviation; SGS, subglottic stenosis; TBM, tracheobronchomalacia; TT, tracheostomy tube.

days of MV, LOHS, etc. of our study were compared with those of other studies and were described in - Table 8.

\section{Discussion}

This prospective study determined the outcomes of postoperative tracheostomy and the predictors of survival.

\section{Mortality}

In our study cohort, the mortality was $29 \%$. Mortality between 25 to $48 \%$ have been described by various authors..$^{1,-6,11-13}$ The various outcome variables in our study population such as mortality, days of endotracheal tube ventilation (DOETTV), days of tracheostomy tube ventilation (DOTTV), number of extubation trials, sepsis, chromosomal abnormality, neurological impairment, VAP, diaphragmatic palsy, chylothorax, tracheomalacia, reexploration, LOHS, discharged with tracheostomy tube in situ, etc. were further compared with the studies conducted by various authors, elaborated in - Table 8 .

\section{Pre and Intraoperative Variables}

Pre and intraoperative variables in our study were found to be statistically similar between the survivors and non-survivors, as described in - Table $\mathbf{2}$. According to Mahida et al, neonatal age $(p=0.04)$, cardiac risk factors $(p=0.04)$, immune system disorders $(p=0.01)$, etc. were associated with major complications, including death, following tracheostomy. ${ }^{14}$ Parisika and colleagues in their study found that hypoalbuminemia $(p=0.04)$, a marker of nutritional status, and duration of MV $(p=0.029)$ prior to tracheostomy were significant associations. ${ }^{15}$ The authors Rosner et al documented that prior cardiac surgery (hazard ratio [HR] 4.7, 95\% CI 1.3-16.4, $p=$ 0.02 ) and cumulative LOHS were associated with decreased survival $(p=0.05)$ following infant tracheostomy. ${ }^{6} \mathrm{~A}$ study by Mastropiero et al showed that the patients who required preoperative MV had a higher mortality rate. ${ }^{1}$

\section{Postoperative Complications}

AKI as a predictor of death was more prevalent in nonsurvivors than survivors following tracheostomy in our study 
Table 7 Comparison of peritracheostomy variables between S and NS following tracheostomy

\begin{tabular}{|c|c|c|c|c|c|c|c|c|c|c|c|c|c|}
\hline \multirow[t]{2}{*}{ SI. no. } & \multirow{2}{*}{$\begin{array}{l}\text { VARM } \\
\pm S D\end{array}$} & \multicolumn{3}{|c|}{ Before tracheostomy } & \multicolumn{3}{|c|}{ Tracheostomy day 1} & \multicolumn{3}{|c|}{ Tracheostomy day 2} & \multicolumn{3}{|c|}{ Tracheostomy day 3} \\
\hline & & $S$ & NS & $p$ & $\mathrm{~S}$ & NS & $p$ & $S$ & NS & $p$ & $S$ & NS & $p$ \\
\hline 1 & $\mathrm{PH}$ & $\begin{array}{l}7.35 \pm \\
0.12\end{array}$ & $\begin{array}{l}7.36 \pm \\
0.14\end{array}$ & 0.87 & $\begin{array}{l}7.42 \pm \\
0.06\end{array}$ & $\begin{array}{l}7.37 \pm \\
0.08\end{array}$ & 0.03 & $\begin{array}{l}7.41 \pm \\
0.06\end{array}$ & $\begin{array}{l}7.39 \pm \\
0.05\end{array}$ & 0.41 & $\begin{array}{l}7.40 \pm \\
0.03\end{array}$ & $\begin{array}{l}7.37 \pm \\
0.06\end{array}$ & 0.05 \\
\hline 2 & PCO2 & $\begin{array}{l}47.6 \pm \\
21.3\end{array}$ & $\begin{array}{l}49.7 \pm \\
39.0\end{array}$ & 0.45 & $\begin{array}{l}37.3 \pm \\
8.9\end{array}$ & $\begin{array}{l}42.8 \pm \\
15.9\end{array}$ & 0.25 & $\begin{array}{l}37.5 \pm \\
9.5\end{array}$ & $\begin{array}{l}38.2 \pm \\
7.3\end{array}$ & 0.55 & $\begin{array}{l}37.5 \pm \\
7.7\end{array}$ & $\begin{array}{l}43.8 \pm \\
14\end{array}$ & 0.18 \\
\hline 3 & $\mathrm{PO} 2$ & $\begin{array}{l}110.1 \pm \\
62.6\end{array}$ & $\begin{array}{l}103.4 \pm \\
118.7\end{array}$ & 0.21 & $\begin{array}{l}123.5 \\
\pm \\
58.3\end{array}$ & $\begin{array}{l}87.6 \pm \\
44.1\end{array}$ & 0.06 & $\begin{array}{l}120.3 \\
\pm \\
60.8\end{array}$ & $\begin{array}{l}97.8 \pm \\
59.7\end{array}$ & 0.28 & $\begin{array}{l}126.3 \\
\pm \\
68.5\end{array}$ & $\begin{array}{l}90.8 \pm \\
52.2\end{array}$ & 0.11 \\
\hline 4 & $\mathrm{BE}$ & $\begin{array}{l}0.11 \pm \\
3.6\end{array}$ & $\begin{array}{l}-1.0 \pm \\
5.3\end{array}$ & 0.3 & $\begin{array}{l}0.34 \pm \\
3.5\end{array}$ & $\begin{array}{l}-1.5 \pm \\
3.5\end{array}$ & 0.17 & $\begin{array}{l}-.06 \pm \\
3.7\end{array}$ & $\begin{array}{l}-0.75 \\
\pm \\
5.67\end{array}$ & 0.63 & $\begin{array}{l}-0.44 \\
\pm \\
3.5\end{array}$ & $\begin{array}{l}0.57 \pm \\
4.77\end{array}$ & 0.56 \\
\hline 5 & $\mathrm{HCO} 3$ & $\begin{array}{l}24.7 \pm \\
3.9\end{array}$ & $\begin{array}{l}24.6 \pm \\
6.8\end{array}$ & 0.94 & $\begin{array}{l}24.2 \pm \\
3.8\end{array}$ & $\begin{array}{l}24.2 \pm \\
3.71\end{array}$ & 0.98 & $\begin{array}{l}23.9 \pm \\
3.7\end{array}$ & $\begin{array}{l}23.4 \pm \\
4.8\end{array}$ & 0.74 & $\begin{array}{l}23.4 \pm \\
3.5\end{array}$ & $\begin{array}{l}25.0 \pm \\
4.6\end{array}$ & 0.26 \\
\hline 6 & $\mathrm{HB}$ & $\begin{array}{l}11.9 \pm \\
1.5\end{array}$ & $\begin{array}{l}11.7 \pm \\
1.2\end{array}$ & 0.68 & $\begin{array}{l}11.9 \pm \\
1.4\end{array}$ & $\begin{array}{l}11.9 \pm \\
1.7\end{array}$ & 0.45 & $\begin{array}{l}12.1 \pm \\
1.3\end{array}$ & $\begin{array}{l}11.7 \pm \\
1.9\end{array}$ & 0.56 & $\begin{array}{l}11.6 \pm \\
1.1\end{array}$ & $\begin{array}{l}11.3 \pm \\
1.3\end{array}$ & 0.47 \\
\hline 7 & PCV & $\begin{array}{l}36.5 \pm \\
3.5\end{array}$ & $\begin{array}{l}35.2 \pm \\
3.6\end{array}$ & 0.3 & $\begin{array}{l}36.1 \pm \\
4.0\end{array}$ & $\begin{array}{l}31.2 \pm \\
10.7\end{array}$ & 0.8 & $\begin{array}{l}37.0 \pm \\
3.3\end{array}$ & $\begin{array}{l}33.8 \pm \\
8.8\end{array}$ & 0.09 & $\begin{array}{l}36.4 \pm \\
3.3\end{array}$ & $\begin{array}{l}33.3 \pm \\
8.2\end{array}$ & 0.1 \\
\hline 8 & $\mathrm{SaO} 2$ & $\begin{array}{l}90.6 \pm \\
13.5\end{array}$ & $\begin{array}{l}86.3 \pm \\
23.4\end{array}$ & 0.4 & $\begin{array}{l}95.2 \pm \\
8.6\end{array}$ & $\begin{array}{l}90.7 \pm \\
8.7\end{array}$ & 0.04 & $\begin{array}{l}93.7 \pm \\
9.9\end{array}$ & $\begin{array}{l}92.4 \pm \\
9.1\end{array}$ & 0.6 & $\begin{array}{l}96.2 \pm \\
4.8\end{array}$ & $\begin{array}{l}92.5 \pm \\
8.3\end{array}$ & 0.08 \\
\hline 9 & HR & $\begin{array}{l}126.3 \pm \\
18.5\end{array}$ & $\begin{array}{l}126.6 \pm \\
20.4\end{array}$ & 0.9 & $\begin{array}{l}129 \pm \\
16.5\end{array}$ & $\begin{array}{l}127.4 \\
\pm \\
17.9\end{array}$ & 0.7 & $\begin{array}{l}126.9 \\
\pm \\
13.7\end{array}$ & $\begin{array}{l}126.0 \\
\pm \\
14.3\end{array}$ & 0.9 & $\begin{array}{l}121.4 \\
\pm \\
16.2\end{array}$ & $\begin{array}{l}127 . \pm \\
16.6\end{array}$ & 0.27 \\
\hline 10 & SBP & $\begin{array}{l}87.8 \pm \\
15.2\end{array}$ & $\begin{array}{l}81.7 \pm \\
17.8\end{array}$ & 0.27 & $\begin{array}{l}89.4 \pm \\
17.3\end{array}$ & $\begin{array}{l}84.7 \pm \\
16.5\end{array}$ & 0.4 & $\begin{array}{l}90.7 \pm \\
14.8\end{array}$ & $\begin{array}{l}83.6 \pm \\
19.8\end{array}$ & 0.2 & $\begin{array}{l}89.7 \pm \\
18.1\end{array}$ & $\begin{array}{l}83.5 \pm \\
16.3\end{array}$ & 0.3 \\
\hline 11 & DBP & $\begin{array}{l}52.6 \pm \\
10.1\end{array}$ & $\begin{array}{l}52.2 \pm \\
13.7\end{array}$ & 0.3 & $\begin{array}{l}56.2 \pm \\
13.3\end{array}$ & $\begin{array}{l}56 \pm \\
17.3\end{array}$ & 0.3 & $\begin{array}{l}58.2 \pm \\
9.8\end{array}$ & $\begin{array}{l}53.4 \pm \\
19.7\end{array}$ & 0.9 & $\begin{array}{l}56 \pm \\
10.0\end{array}$ & $\begin{array}{l}52.3 \pm \\
10.6\end{array}$ & 0.8 \\
\hline 12 & RA & $\begin{array}{l}11.5 \pm \\
5.2\end{array}$ & $\begin{array}{l}11.3 \pm \\
5.3\end{array}$ & 0.6 & $\begin{array}{l}12.3 \pm \\
5.7\end{array}$ & $\begin{array}{l}12.9 \pm \\
5.3\end{array}$ & 0.9 & $\begin{array}{l}11.8 \pm \\
4.7\end{array}$ & $\begin{array}{l}12.8 \pm \\
6.7\end{array}$ & 0.6 & $\begin{array}{l}10.6 \pm \\
5.4\end{array}$ & $\begin{array}{l}12.9 \pm \\
6.5\end{array}$ & 0.2 \\
\hline 13 & LAC & $\begin{array}{l}1.2 \pm \\
0.9\end{array}$ & $\begin{array}{l}2.4 \pm \\
3.0\end{array}$ & 0.5 & $\begin{array}{l}1.1 \pm \\
0.79\end{array}$ & $\begin{array}{l}2.1 \pm \\
3.5\end{array}$ & 0.3 & $\begin{array}{l}0.99 \pm \\
0.88\end{array}$ & $\begin{array}{l}3.2 \pm \\
3.7\end{array}$ & 0.01 & $\begin{array}{l}1.0 \pm \\
0.5\end{array}$ & $\begin{array}{l}2.4 \pm \\
2.1\end{array}$ & 0.01 \\
\hline 14 & RBS & $\begin{array}{l}114 \pm \\
38.1\end{array}$ & $\begin{array}{l}106 \pm \\
21.1\end{array}$ & 0.5 & $\begin{array}{l}115 \pm \\
27.4\end{array}$ & $\begin{array}{l}156.1 \\
\pm \\
140.1\end{array}$ & 0.13 & $\begin{array}{l}110.9 \\
\pm \\
27.3\end{array}$ & $\begin{array}{l}107.0 \\
\pm \\
26.8\end{array}$ & 0.6 & $\begin{array}{l}109.9 \\
\pm \\
27.9\end{array}$ & $\begin{array}{l}137.0 \\
\pm \\
57.8\end{array}$ & 0.04 \\
\hline 15 & VIS & $\begin{array}{l}41.6 \pm \\
126\end{array}$ & $\begin{array}{l}19.1 \pm \\
14.6\end{array}$ & 0.9 & $\begin{array}{l}85.6 \pm \\
374\end{array}$ & $\begin{array}{l}21.3 \pm \\
15.3\end{array}$ & 0.3 & $\begin{array}{l}16.1 \pm \\
15.2\end{array}$ & $\begin{array}{l}20.4 \pm \\
15.7\end{array}$ & 0.36 & $\begin{array}{l}11.1 \pm \\
9.6\end{array}$ & $\begin{array}{l}18.2 \pm \\
14.8\end{array}$ & 0.15 \\
\hline 16 & CFB & $\begin{array}{l}-79.2 \pm \\
181\end{array}$ & $\begin{array}{l}-161 \pm \\
345\end{array}$ & 0.7 & $\begin{array}{l}-106 \\
\pm \\
190\end{array}$ & $\begin{array}{l}-199 \\
\pm \\
367\end{array}$ & 0.6 & $\begin{array}{l}-136 \\
\pm \\
214\end{array}$ & $\begin{array}{l}-217 \\
\pm \\
395\end{array}$ & 0.6 & $\begin{array}{l}-163 \\
\pm \\
235\end{array}$ & $\begin{array}{l}-231 \\
\pm \\
423\end{array}$ & 0.8 \\
\hline 17 & PT & $\begin{array}{l}15.72 \pm \\
1.24\end{array}$ & $\begin{array}{l}16.03 \pm \\
1.40\end{array}$ & 0.6 & & & & & & & & & \\
\hline 18 & aPTT & $\begin{array}{l}32.7 \pm \\
2.78\end{array}$ & $\begin{array}{l}43.41 \pm \\
9.93\end{array}$ & 0.006 & & & & & & & & & \\
\hline 19 & INR & $\begin{array}{l}1.32 \pm \\
0.12\end{array}$ & $\begin{array}{l}2.26 \pm \\
1.59\end{array}$ & 0.09 & & & & & & & & & \\
\hline 20 & PLT & $\begin{array}{l}118.10 \\
\pm \\
99.42\end{array}$ & $\begin{array}{l}74.81 \pm \\
64.87\end{array}$ & 0.009 & & & & & & & & & \\
\hline 21 & $\begin{array}{l}\text { BLD, } n \\
(\%)\end{array}$ & $0(0)$ & $3(25)$ & 0.02 & & & & & & & & & \\
\hline 22 & PEEP & $\begin{array}{l}4.79 \pm \\
0.77\end{array}$ & $\begin{array}{l}4.75 \pm \\
0.45\end{array}$ & 0.7 & & & & & & & & & \\
\hline
\end{tabular}

Abbreviations: aPTT, activated partial thromboplastin time in seconds; BE, base excess in meq/liter; BLD, bleeding from TT site: no bleeding; CFB, cumulative fluid balance in $\mathrm{mL} / \mathrm{kg}$; DBP, diastolic blood pressure in $\mathrm{mm} \mathrm{Hg} ; \mathrm{HB}$, hemoglobin in $\mathrm{g} / \mathrm{dl} ; \mathrm{HCO}$, bicarbonate in meq/l; HR, heart rate in per minute; INR, international normalized ratio; LAC, lactate in mmol/l; $\mathrm{M}+/$-SD, mean $+/$ - standard deviation; $n$, number; NS, nonsurvivors; $p, p$ value; PCO2, partial pressure of CO2 in mm Hg; PCV, hematocrit in \%; PLT, platelet count in ${ }^{*} 1000 / \mathrm{mL}$; PO2, partial pressure of $\mathrm{O} 2$ in $\mathrm{mm} \mathrm{Hg}$; PT, prothrombin time in seconds; RA, right atrial pressure in mm Hg; RBS, random blood sugar mg/dl; S, survivors; SaO2, saturation of o2 in \%; SBP, systolic blood pressure in mm $\mathrm{Hg}$; TT, tracheostomy; VAR, variable; VIS, vasoactive inotropic score. 
Table 8 Comparison of different variables of our study population with similar studies conducted by other authors

\begin{tabular}{|c|c|c|c|c|c|c|}
\hline SI. no. & Variables & $\begin{array}{l}\text { Our study } \\
N=1084\end{array}$ & $\begin{array}{l}\text { Suygi et al }{ }^{11} \\
N=1450\end{array}$ & $\begin{array}{l}\text { Alibrahim et } \text { al }^{12} \\
N=3344\end{array}$ & $\begin{array}{l}\text { Cotts et } \mathrm{al}^{5} \\
\mathrm{~N}=4503\end{array}$ & $\begin{array}{l}\text { Hosokote et } \mathrm{al}^{4} \\
\mathrm{~N}=1370\end{array}$ \\
\hline 1 & $\begin{array}{l}\text { Tracheostomy incidence, } n \\
(\%)\end{array}$ & $41(3.7)$ & $18(1.2)$ & $16(0.5)$ & $59(1.3)$ & $37(2.7)$ \\
\hline 2 & Mortality, n (\%) & $12(29)$ & $6(33.3)$ & $6(37.5)$ & $13(22)$ & $15(40.5)$ \\
\hline 3 & DOETTV in days (mean \pm SD) & $13.04 \pm 9.67$ & $41.0 \pm 12.9$ & $60.4 \pm 9.8$ & 36 (median) & 30 (median) \\
\hline 4 & $\begin{array}{l}\text { No. of extubation attempts } \\
\text { (mean } \pm \text { SD) }\end{array}$ & $1.09 \pm 0.9$ & $4.2 \pm 0.8$ & 1.25 & & 1 (median) \\
\hline 5 & Sepsis, $n(\%)$ & $13(31.7)$ & $14(78)$ & $\begin{array}{l}\text { Pre-TT } 9 \text { (56) } \\
\text { Post-TT } 6 \text { (38) }\end{array}$ & & \\
\hline 6 & $\begin{array}{l}\text { Chromosomal abnormality, } \\
n(\%)\end{array}$ & $5(12)$ & $3(17)$ & $3(18.8)$ & $24(40)$ & $12(32)$ \\
\hline 7 & $\begin{array}{l}\text { Neurological impairment, } \\
n(\%)\end{array}$ & $12(29)$ & $2(11)$ & & & $10(27)$ \\
\hline 8 & Total VAP, $n(\%)$ & $13(31.7)$ & $2(11)$ & & & \\
\hline 9 & Pretracheostomy VAP, $n$ (\%) & $8(19.5)$ & & $8(50)$ & & \\
\hline 10 & Posttracheostomy VAP, $n$ (\%) & $5(12.2)$ & & $0(0)$ & & \\
\hline 9 & $\begin{array}{l}\text { Diaphragmatic paralysis, } n \\
(\%)\end{array}$ & $1(2.4)$ & $3(17)$ & $4(25)$ & & $13(35)$ \\
\hline 10 & Chylothorax, $n(\%)$ & $5(12.2)$ & $2(11)$ & $2(12.5)$ & & $3(8)$ \\
\hline 11 & Tracheomalacia, $n(\%)$ & $1(2.4)$ & $1(5.5)$ & & $14(23.7)$ & $18(49)$ \\
\hline 12 & $\begin{array}{l}\text { Discharged with tracheos- } \\
\text { tomy in situ, } n(\%)\end{array}$ & $7(17.1)$ & $8(44)$ & $3(18.75)$ & $22(37.2)$ & \\
\hline 13 & DOTTV in days (mean \pm SD) & $21.38 \pm 19.14$ & & $14.5 \pm 4.8$ & & \\
\hline 14 & LOHS in days (mean \pm SD) & $54.95 \pm 36.9$ & & & 62 (med) & \\
\hline 15 & Reexploration, $n(\%)$ & $24(58.5)$ & & & & $21(57)$ \\
\hline
\end{tabular}

Abbreviations: DOETTV, days of endotracheal tube ventilation; DOTTV, days of tracheostomy tube ventilation; med, median; $n$, number; N, number of index pediatric cardiac surgery; $\mathrm{N}$, number of children tracheostomized; SD, standard deviation; VAP, ventilator-associated pneumonia.

$(p<0.001)$. Similar to our observation, Waltz et al had observed a high blood urea levels in association with mortality $(p=0.0001) .{ }^{16} \mathrm{~A}$ study by Deng et al noticed that AKI was significantly associated with tracheostomy $(p<0.001)$ and mortality $(p<0.001) .{ }^{17}$

In our study, we noticed that the nonsurvivors had more days with open sternum than survivors $(p=0.04)$. We infer that this significant association might be because of higher chances of infection and sepsis due to open sternum. The implication of delayed SC had a bearing on postoperative sepsis and death.

Mahida and colleagues in their study noticed that there is a statistically significant association between CNS injury in the form of intracranial hemorrhage and mortality $(p=$ 0.048)..$^{14}$ Mastropietro et al described several major adverse events (mechanical circulatory support, cardiac arrest, additional cardiovascular procedures), which occurred more frequently in the patients with tracheostomy who ultimately died than those who survived. ${ }^{1}$

\section{Postoperative Infection}

We found that there was a statistically significant association between nonsurvivors and high-serum CRP. According to Bhattacharya et al, a simple, easily accessible marker like CRP was an important determinant of mortality $(p=0.0001) .^{18}$ We routinely tested the CRP levels as a biomarker of inflammation and infection in our unit, as it was economically viable than others like procalcitonin. We used procalcitonin more judiciously while taking decisions on escalating/deescalating antibiotic therapy while treating HAI and sepsis. We did not notice mortality benefit with procalcitonin estimation. Although procalcitonin has been considered as a marker to regulate the antibiotics during course of sepsis management, it is a bit controversial in patients undergoing cardiopulmonary bypass. ${ }^{19}$

In a study by Mastropietro et al, sepsis occurred more frequently in the patients with tracheostomy who ultimately died. ${ }^{1}$ In contrast to the results of the study by Mastropietro, Nseir and colleagues in their case control study demonstrated that patients who developed VAP had higher ICU mortality $(p=0.001)$ than the non-VAP population and tracheotomy was independently associated with decreased risk of VAP $(p=0.003) .{ }^{20}$ On similar lines, our study did not find any association with infection, sepsis and mediastinitis in terms of survival in tracheostomized children. A similar finding was shown by Waltz et al. ${ }^{16}$ However, Curtis and colleagues had shown a higher association of SSI in tracheostomy patients in their study. ${ }^{21}$ 


\section{Postoperative Nutrition}

In our study, we found that there was a statistically significant association between tracheostomy-associated mortality and the requirement of TPN ( $p=0.005)$ as well as prevalence of FI ( $p=0.02$ ). We concluded that being able to supplement the daily required energy and protein enterally was associated with better survival. A similar observation was made by Strak van Schijndel et $\mathrm{a}^{22}$ in their study-patients who reached their nutritional goals compared with those who did not showed a HR of 0.199 for ICU mortality (CI 0.048-0.831; $p=0.027$ ), a HR of 0.079 for 28-day mortality (CI 0.013 $0.467 ; p=0.005$ ), and a HR of 0.328 for hospital mortality (CI 0.113-0.952; $p=0.04)$.

\section{Peritracheostomy Variables}

In this study, we noticed that higher aPTT levels $(p=0.006)$, lower platelet count before tracheostomy $(p=0.009)$, and higher bleeding complications ( $p=0.02$ ) after tracheostomy were associated with nonsurvivors and statistically significant. This was because of inflammation, infection, and transfusion-related coagulation derangement, which happened more with the nonsurvivor group.

We noted that a lower $\mathrm{pH}$ was significantly associated with the nonsurvivor group at various time points (day 1 after tracheostomy $p=0.03$, day 3 after tracheostomy $p=$ $0.05)$. According to the study by Walts et al, lower $\mathrm{pH}$ was a significantly associated with mortality $(p=0.004){ }^{16}$.

In our study, survivors were found to have statistically significant lower lactate levels at various time points (day2 after tracheostomy; $p=0.01$; day 3 after tracheostomy, $p=0.01$ ). Irrespective of its origin, hyperlactatemia and its persistence have been demonstrated to be an early indicator of worse outcome in cardiac surgical patients. ${ }^{23}$ Rocha et al recently evaluated lactate trend in their postoperative pediatric population and found that increased lactate levels in the third hour appeared to be suggestive of worse prognosis. ${ }^{24}$ Our findings were in concordance with the above study.

We noted that the peak RBS levels on day 3 after tracheostomy were significantly higher in the nonsurvivors $(p=0.04)$. According to the study by Patki and colleagues, the mortality among hyperglycemic children was significantly higher than nonhyperglycemic children. ${ }^{25}$

\section{Study Limitations}

The observational nature of this study precluded us from drawing any causality between the survival and perioperative risk factors and other morbidities. Furthermore, we did not perform a formal cost analysis, but reductions in ICU and hospital LOS was always associated with considerable cost savings and resource optimization. Finally, the number of patients who underwent tracheostomy is low when compared with other studies. Another important lacuna of this study was that we did not evaluate the intermediate and long-term outcomes.

\section{Conclusion}

Our study demonstrated a survival of 71\% among the study cohort who underwent tracheostomy following congenital cardiac repairs. Shorter duration of open sternum, lesser incidence of AKI, shorter days on TPN and FI, lesser aPTT, lower posttracheostomy levels of lactate, RBS and bleeding from tracheostomy site, as well as a higher pretracheostomy platelet count and posttracheostomy $\mathrm{SaO} 2$ were the predictors of survival in these children.

\section{Funding}

The authors declare that no funds were received from any source for conducting this project.

\section{Conflict of Interest}

None declared.

\section{References}

1 Mastropietro CW, Benneyworth BD, Turrentine M, et al. Tracheostomy after operations for congenital heart disease: an analysis of the society of thoracic surgeons congenital heart surgery database. Ann Thorac Surg 2016;101(6):2285-2292

2 LoTempio MM, Shapiro NL. Tracheotomy tube placement in children following cardiothoracic surgery: indications and outcomes. Am J Otolaryngol 2002;23(6):337-340

3 Rossi AF, Fishberger S, Hannan RL, et al. Frequency and indications for tracheostomy and gastrostomy after congenital heart surgery. Pediatr Cardiol 2009;30(3):225-231

4 Hoskote A, Cohen G, Goldman A, Shekerdemian L. Tracheostomy in infants and children after cardiothoracic surgery: indications, associated risk factors, and timing. J Thorac Cardiovasc Surg 2005;130(4):1086-1093

5 Cotts T, Hirsch J, Thorne M, Gajarski R. Tracheostomy after pediatric cardiac surgery: frequency, indications, and outcomes. J Thorac Cardiovasc Surg 2011;141(2):413-418

6 Rosner E, Mastropietro CW. Prior cardiac surgery is independently associated with decreased survival following infant tracheostomy. Respir Care 2015;60(1):47-55

7 Peinado DMC, Bedrinana JIC. Comparison of NCHS-1977, CDC-2000 and WHO-2006 Nutritional Classification in 32 to 60 month-old Children in the Central Highlands of Peru (1992-2007. Univers J Public Health 2013;1(3):143-149

8 Kasiske B. Eckardt K. Kidney Disease Improving Global Outcomes (KDIGO) Acute Kidney Injury Work Group. KDIGO Clinical Practice Guideline for Acute Kidney Injury. Kidney Inter Suppl 2012;2:1-138

9 Singer M, Deutschman CS, Seymour CW, et al. The third international consensus definitions for sepsis and septic shock (Sepsis-3. JAMA 2016;315(8):801-810

10 Centers for Disease Control and Prevention/National Healthcare Safety Network. CDC/NHSN surveillance definitions for specific types of infections. Available at: https:// www.cdc.gov/nhsn/pdfs/pscmanual/2020pscnosinfdef_current.pdf. Accessed May 19, 2020

11 Suygi M, Ozturk E, Ozden O, et al. Evaluation of tracheostomy patients in a pediatric cardiac intensive care unit: our five-year single-center experiences. Turk Gogus Kalp Dama 2016;24(2):227-232

12 Alibrahim IJ, Kabbani MS, Abu-Sulaiman R, Al-Akhfash A, Mazrou KA. Outcome of tracheostomy after pediatric cardiac surgery. J Saudi Heart Assoc 2012;24(3):163-168 
13 Enc Y, Ayidemer N, Bicer Y, et al. Indications and results of tracheostomy in pediatric postoperative intensive care unit. Turkish J Thorac Cardiovasc Surg 2008;16(1):24-28

14 Mahida JB, Asti L, Boss EF, et al. Tracheostomy placement in children younger than 2 years: 30 -day outcomes using the National Surgical Quality Improvement Program Pediatric. JAMA Otolaryngol Head Neck Surg 2016;142(3):241-246

15 Parsikia A, Goodwin M, Wells Z, et al. Prognostic indicators for early mortality after tracheostomy in the intensive care unit. J Surg Res 2016;206(1):235-241

16 Walts PA, Murthy SC, Arroliga AC, et al. Tracheostomy after cardiovascular surgery: an assessment of long-term outcome. J Thorac Cardiovasc Surg 2006;131(4):830-837

17 Deng Jie, Yuan Ruibin, Chi, et al. The incidence, risk factors and outcomes of postoperative acute kidney injury in neurosurgical critically ill patients. Scientific Reports 2017;7:4245

18 Bhattacharya B, Prashant A, Vishwanath P, Suma MN, Nataraj B. Prediction of outcome and prognosis of patients on mechanical ventilation using body mass index, SOFA score, C-Reactive protein, and serum albumin. Indian J Crit Care Med 2011;15(2):82-87

19 Dai X, Fu C, Wang C, et al. The impact of tracheotomy on levels of procalcitonin in patients without sepsis: a prospective study. Clinics (São Paulo 2015;70(9):612-617
20 Nseir S, Di Pompeo C, Jozefowicz E, et al. Relationship between tracheotomy and ventilator-associated pneumonia: a case control study. Eur Respir J 2007;30(2):314-320

21 Curtis JJ, Clark NC, McKenney CA, et al. Tracheostomy: a risk factor for mediastinitis after cardiac operation. Ann Thorac Surg 2001;72(3):731-734

22 Strack van Schijndel RJ, Weijs PJM, Koopmans RH, Sauerwein HP, Beishuizen A, Girbes AR. Optimal nutrition during the period of mechanical ventilation decreases mortality in critically ill, long-term acute female patients: a prospective observational cohort study. Crit Care 2009;13(4):R132

23 Ranucci M, De Toffol B, Isgrò G, Romitti F, Conti D, Vicentini M. Hyperlactatemia during cardiopulmonary bypass: determinants and impact on postoperative outcome. Crit Care 2006;10(6):R167

24 Rocha TS, Silveira AS, Botta AM, Ricachinevsky CP, Dalle Mulle L, Nogueira A. Serum lactate as mortality and morbidity marker in infants after Jatene's operation. Rev Bras Cir Cardiovasc 2010;25(3):350-358

25 Patki VK, Chougule SB. Hyperglycemia in critically ill children. Indian J Crit Care Med 2014;18(1):8-13 